\title{
Jones calculus analysis of single-mode fiber Sagnac reflector
}

\author{
T. A. Birks and P. Morkel
}

\begin{abstract}
It is postulated that stray birefringence degrades the performance of the single-mode fiber Sagnac reflector. An expression for the birefringence dependence of reflector performance is derived using the Jones calculus and is used as the basis for a theoretical model of the spectral response of a real Sagnac reflector. The experimental behavior of the reflector with (1) different levels of stress applied to the fiber loop and (2) different lengths of highly birefringent fiber spliced into the loop are compared with the model. This comparison confirms the initial postulate.
\end{abstract}

\section{Introduction}

As more single-mode fiber compatible components are being developed, their interconnection in the form of fiber circuits is increasingly finding new applications. ${ }^{1-3}$ In general such circuits have been constructed assuming ideal polarization behavior of both the components and fiber leads ${ }^{2}$ with the result that the nonideal behavior, which imposes limitations on the performance of real circuits, can yield unexpected circuit responses. A thorough treatment of birefringence effects in fiber circuits is required if the technology is to be exploited to the full.

A simple example of a fiber circuit is the Sagnac reflector which has been used as a feedback element in fiber lasers. ${ }^{2}$ Such a reflector can be made by splicing together the output ports of a $3-\mathrm{dB}$ power-splitting $2 \times$ 2 fiber coupler yielding a Sagnac interferometer as shown in Fig. 1. In the ideal case, light entering the input port is split by the coupler into two counterpropagating waves of equal amplitude which propagate around the loop. Because both waves travel the same optical path, constructive interference is assured when the waves reenter the coupler. All the light is then reflected back into the input port, with the reflectivity limited only by coupler, fiber, and splice losses. In

The authors are with University of Southampton, Department of Electronics \& Computer Science, Optical Fibre Group, Southampton S09 5NH, U.K.

Received 19 October 1987.

0003-6935/88/153107-07\$02.00/0.

(C) 1988 Optical Society of America. practice it is found that the performance of the reflector is degraded from this ideal.

In this paper, it is postulated that this degradation is due to stress-induced birefringence in the fiber loop. This birefringence, in general, destroys the constructive interference required for the successful operation of the reflector. Such birefringences will always set a limit on the performance of fiber circuitry.

We use a Jones calculus ${ }^{4}$ approach for analyzing the Sagnac reflector, which can take account of the birefringences of components and fiber leads. The results of the analysis are compared with the experimental results for a Sagnac reflector where the loop of the reflector is subjected to large strains and where the loop contains a known length of highly birefringent fiber. This comparison confirms our postulate that the degradation in reflector performance is due to stress-induced birefringence.

\section{Analysis of the Sagnac Reflector}

The light wave at any point in the circuit is represented by its Jones vector:

$$
\mathbf{E}=\left(\begin{array}{c}
E_{x} \\
E_{y}
\end{array}\right) .
$$

To apply the Jones calculus we need to identify the Jones matrix for each sequential element of the optical path. The effect of each circuit element will then be represented by a $2 \times 2$ matrix operating on the Jones vector $\mathbf{E}$. The effect of the whole circuit will then be described by combining these matrices according to the rules of the Jones calculus. ${ }^{4}$ In the case of the Sagnac reflector there are three elements, with reference to Fig. 1:

(1) The light wave passes from the input port 1 of the coupler and is split between the output ports 3 and 4. 
(2) The two light waves propagate around the loop in opposite directions.

(3) The light waves reenter the coupler through ports 3 and 4 where they recombine and return to the input ports 1 and 2.

We now deduce the Jones matrices for these three elements; to simplify the analysis we neglect the effects of coupler, fiber, and splice losses.

\section{A. Coupler in the Forward Direction}

The single-mode directional coupler is characterized by its coupling coefficient $C$ and its coupling length $l_{c}$. If we assume that the coupling coefficient is independent of polarization, for an electric field

$$
\mathbf{E}_{1}=\left(\begin{array}{l}
E_{1 x} \\
E_{1 y}
\end{array}\right)
$$

entering port 1, the output electric fields can be expressed as

$$
\begin{aligned}
& \mathbf{E}_{3}=\cos \left(C l_{c}\right) M_{c} \mathbf{E}_{1}, \\
& \mathbf{E}_{4}=i \sin \left(C l_{c}\right) M_{c} \mathbf{E}_{1},
\end{aligned}
$$

where $M_{c}$ is a $2 \times 2$ Jones matrix representing any birefrigence in the coupling region.

\section{B. Effects of the Fiber Loop}

In general, we can regard the loop as being elliptically birefringent and without Faraday rotation.

The loop has two different Jones matrices depending on the direction of propagation around the loop. If $\alpha$ is the azimuthal angle of the major axis of the ellipse of birefringence of the loop, with reference to the coupler axes as defined in Fig. 2, the Jones matrix for light traveling from port 3 to port 4 is ${ }^{4}$

$$
\begin{aligned}
M(\alpha) & =\left(\begin{array}{ll}
\cos (\delta / 2)+i \cos (2 R) \sin (\delta / 2) & i \sin (\delta / 2) \sin (2 R) \exp (i \gamma) \\
i \sin (\delta / 2) \sin (2 R) \exp (-i \gamma) & \cos (\delta / 2)-i \cos (2 R) \sin (\delta / 2)
\end{array}\right) \\
& =\left(\begin{array}{cc}
A & B \\
-B^{*} & A^{*}
\end{array}\right)
\end{aligned}
$$

where ${ }^{5}$

$$
\tan (2 \gamma)=\tan (2 R) \cos (\gamma),
$$

$\delta, R, \gamma$ are described below and are as defined in Ref. 4 , and $A^{*}$ is the complex conjugate of $A$ etc.

The term $\delta$ is the phase retardance, which can be related to the number of beat lengths in the loop through

$$
\cdot \delta=2 \pi N,
$$

where $N$ corresponds to $\delta_{c}$ of Ref. 6 and is not necessarily an integer. Note that beat length here refers to the elliptical birefringence beat length. The terms $R$ and $\gamma$ combine to specify the ellipticity and angle of birefringence.

Because of the $180^{\circ}$ direction change induced by the loop, the Jones matrix for the light traveling from port 4 to port 3 is characterized by the azimuthal angle of $-\alpha$ and is given by

$$
M(-\alpha)=\left(\begin{array}{cc}
A & B^{*} \\
-B & A^{*}
\end{array}\right)
$$



Fig. 1. Construction of the fiber Sagnac reflector showing the 3-dB coupler with the port numbering as used in the text.

which can be derived from the definitions of $\delta, R, \gamma$, and the relationship in eq. (4).

Hence, on traversing the loop, the waves reentering the coupler will be

$$
\begin{aligned}
& \mathbf{E}_{3}^{\prime}=M(-\alpha) \mathbf{E}_{4}, \\
& \mathbf{E}_{4}^{\prime}=M(\alpha) \mathbf{E}_{3},
\end{aligned}
$$

where the prime refers to the returning waves.

As footnotes are not used in this journal one should note here parenthetically that the birefringence of an optical element is defined by a mapping of input polarization states to output polarization states. The mathematical representation of this mapping will depend on the systems of axes used to express these input and output polarizations. Normally the output wave propagates in the same direction as the input wave, and the same set of axes can be used to express the polarizations of both; but if there is a change in wave
(3)

direction, two different sets of axes are necessary to describe the input and output polarizations. If the $z$ axis at any point in the circuit is defined to lie parallel to the direction of propagation of the wave at that point, there remains an ambiguity as to the orientation of the $x$ and $y$ axes. For convenience, in this paper we define the $x$ axis at the coupler ports to coincide with the upward normal to the plane of the coupler at all times; the $y$ axis is then defined so that $x, y$, and $z$ form a right-handed set of axes.

In Fig. 2, $(x, y, z)$ are the axes used for waves leaving ports 3 and 4 , and $\left(x^{\prime}, y^{\prime}, z^{\prime}\right)$ are the axes used for waves reentering ports 3 and 4 . If the ellipse of the net loop birefringence is inclined at an angle $\alpha$ for a wave leaving port 3 with reference to axes $(x, y, z)$, it will be inclined at the same angle $\alpha$ for the wave entering port 4 with reference to axes $\left(x^{\prime}, y^{\prime}, z^{\prime}\right)$. However, this ellipse will be inclined at an angle $-\alpha$ for a wave leaving port 4 with reference to axes $(x, y, z)$; and, alt ${ }_{1+}$ ungh the character of the birefringence (phase retardance, ellipticity, and handedness) will otherwise be identical for 


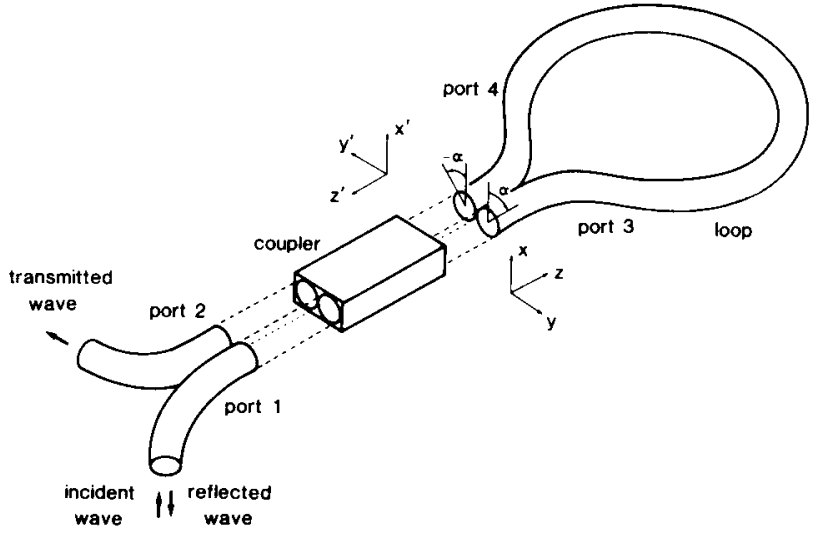

Fig. 2. Schematic of the reflector showing the systems of axes required to express the loop birefringence.

waves leaving either port, for the light wave leaving port 4 it will be characterized by an azimuthal angle $-\alpha$ instead of $\alpha$.

Note that birefringence for purposes of this paper may include a rotation due to geometric effects ${ }^{7}$ as well as material birefringence.

\section{Coupler in the Reverse Direction}

If $\mathrm{M}_{c}^{\prime}$ is the Jones matrix representing the birefringence in the reverse direction through the coupling region, the

$$
\begin{aligned}
& \mathbf{E}_{1}^{\prime}=\mathbf{M}_{c}^{\prime}\left[\cos \left(C l_{c}\right) \mathbf{E}_{3}^{\prime}+i \sin \left(C l_{c}\right) \mathbf{E}_{4}^{\prime}\right], \\
& \mathbf{E}_{2}^{\prime}=\mathbf{M}_{c}^{\prime}\left[i \sin \left(C l_{c}\right) \mathbf{E}_{3}^{\prime}+\cos \left(C l_{c}\right) \mathbf{E}_{4}^{\prime}\right] .
\end{aligned}
$$

\section{Total Effect of the Reflector}

On combining Eqs. (1), (2), and (7)-(10) we find that

$$
\mathbf{E}_{1}^{\prime}=i \sin \left(2 C l_{c}\right) \mathbf{M}_{c}^{\prime}[M(\alpha)+M(-\alpha)] / 2 \mathbf{M}_{c} \mathbf{E}_{1} .
$$

The reflectivity $\rho$ of the reflector is given by the ratio of the reflected power to the incident power in the input port 1:

$$
\rho=\frac{\mathbf{E}_{1}^{\dagger}{ }^{\dagger} \mathbf{E}_{1}^{\prime}}{\mathbf{E}_{1}^{\dagger} \mathbf{E}_{1}},
$$

where the dagger ${ }^{\dagger}$ indicates the Hermitian adjoint. From Eqs. (3), (6), and (11) and by using the unitary property of Jones matrices representing loss-free elements, this equation reduces to

$$
\rho=\sin ^{2}\left(2 C l_{c}\right)\left[1-\sin ^{2}(\pi N) \sin ^{2}(2 R) \cos ^{2}(\gamma)\right] .
$$

The transmittivity $\tau$ of the reflector is given by the ratio of the transmitted power in the output port 2 to the incident power in the input port 1.

$$
\tau=\frac{\mathbf{E}_{2}{ }^{\dagger} \mathbf{E}_{2}^{\prime}}{\mathbf{E}_{1}^{\dagger} \mathbf{E}_{\mathrm{l}}} .
$$

It can readily be shown that

$$
\tau=1-\rho .
$$

It is important to note that the reflectivity is independent of the input polarization.

E. Implications of Eq. (13)

In the absence of birefringence in the fiber loop, Eq (13) reduces to a reflector response determined solely by the coupler characteristics, namely,

$$
\rho=\sin ^{2}\left(2 C l_{c}\right)
$$

Since $C$ is a function of wavelength ${ }^{8}$ the reflected power will also vary with wavelength. A reflectivity of unity occurs at the wavelengths for which $C l_{c}=(1+$ $2 m$ ) $\pi / 4$ (integer $m$ ), which are the $3-\mathrm{dB}$ splitting points of the coupler. ${ }^{2}$

The factor $\left[1-\sin ^{2}(\pi N) \sin ^{2}(2 R) \cos ^{2}(\gamma)\right]$ represents the degradation in reflector performance caused by the loop birefringence.

The $\sin ^{2}(\pi N)$ term is related to the amount of birefringence in the loop and as such will also contribute a wavelength-dependent response. ${ }^{6,9}$ This term fluctuates between 0 and 1 depending on the number of beat lengths in the loop; i.e., if $\sin ^{2}(\pi N)=0$, then $N$ is an integer (full wave retardance), and if $\sin ^{2}(\pi N)=1$, then $2 N$ is an odd integer (halfwave retardance). The factor $\sin ^{2}(2 R) \cos ^{2}(\gamma)$ depends on the type of birefringence in the loop. It is difficult to quantify, but we can assume that it should not have a strong wavelength response. This is the case when one contribution to the overall birefringence predominates. This factor determines the depth of modulation of the degradation present in the reflector and shall be referred to as the degradation amplitude (DA).

\section{Experiment}

\section{A. Experimental Performance of the Sagnac Reflector}

To check the validity of the theory so far presented we constructed a Sagnac reflector using a $2 \times 2$ coupler with nominal $3-\mathrm{dB}$ splitting at $820 \mathrm{~nm}$. The spectral transmittance of the reflector was measured with no external disturbance applied and then with increasing levels of strain applied to the loop. To set a control on the experiment we also inserted sections of known length of highly birefringent bow-tie fiber into the loop. We use these to confirm conclusively that it is the birefringence in the loop that degrades the performance of the reflector.

The measurement system produced results in the form of graphs of

$$
T=-10 \log _{10}(\tau)
$$

against wavelength: hence a maximum in a graph corresponds to a maximum in reflector performance.

\section{B. Ideal Response as Determined from Coupler Characteristics}

The ideal transmittance $\tau$ of the Sagnac reflector is determined from the measured characteristics of the 2 $\times 2$ coupler used in the experiment and assuming no birefringence in the fiber loop. The response $T$ for this case is plotted in Fig. 3 and tends to $\infty$ at the $3-\mathrm{dB}$ splitting point of $820 \mathrm{~nm}$. 




Fig. 3. Ideal spectral transmission $T$ of the reflector using the measured coupler characteristics and assuming no loop birefringence.

\section{Actual Response of Sagnac Reflector}

\section{No Strain}

Figure 4(a) shows the response of the reflector lying unrestrained on the bench with no deliberately imposed external stresses but also with no special care taken to reduce incidental deformation. The performance is clearly much degraded from the ideal performance predicted in Fig. 3 .

\section{With Applied Strain}

Strains were applied to the loop by subjecting the fiber to pressure in a $G$-clamp. The type of response depended very much on the extent of pressure applied. Figures 4(b) and (c) show the responses for lesser and greater pressure, respectively. In each case the response lies under the ideal envelope as drawn in Fig. 3. The greater the strain the shorter is the period of the degrading modulation. The peak at $820 \mathrm{~nm}$ in each case is simply due to the fortuitous coincidence of modulation period and coupler response.

\section{Response with HiBi Fiber Spliced into the Loop}

Different known lengths of highly birefringent bowtie fiber ${ }^{9}(\mathrm{ND} 63$, beat length $=4 \mathrm{~mm}$ at $633 \mathrm{~nm})$ were spliced into the loop, and again the transmission was measured. Figures 5(a)-(c) show the response for lengths of $5,10.5$, and $34 \mathrm{~cm}$ of $\mathrm{HiBi}$, respectively. These responses also lie under the ideal envelope of Fig. 3 , and the period of the degrading modulation shows a marked decrease with increase in the length of the inserted $\mathrm{HiBi}$ fiber. To illustrate the sensitivity of the loop to external effects, Fig. 5(d) shows the response for the same loop as Fig. 5(c) but with the loop configuration on the bench lightly altered.

\section{E. Sensitivity to Input Polarization}

It was confirmed that the performance of the device was in each case independent of the input polarization state by inserting a polarizer at the coupler input port and taking measurements for different polarizer orientations.

\section{Testing of Theory}

In this section we test the theory presented in Sec. II to confirm our postulate that the degradation in the performance of the Sagnac reflector is due to the birefringence of the loop.

First, we need to model the wavelength response of the coupler. The coupling coefficient $C$ is assumed to have a $\lambda^{5 / 2}$ wavelength dependence as would be expected for a weakly fused coupler. ${ }^{8}$ The coupling length $l_{c}$ is chosen so that the coupler has its first 3-dB power splitting point at $820 \mathrm{~nm}$. The graph in Fig. 6 shows the ideal transmission response for such a coupler, and its similarity with Fig. 3 confirms the suitability of the above assumptions.

Second, we need to model the wavelength response of the birefringence. Here we make the usual assumption that the birefringence varies inversely with $\lambda$ so that ${ }^{6,9}$

$$
N=N_{0} \lambda_{0} / \lambda,
$$

where $N_{0}$ is specified at $\lambda_{0}$.

Using the above wavelength dependences and assuming that the degradation amplitude is wavelength independent we calculate the transmittivity of the reflector from Eqs. (13) and (15).

The results for different numbers of beat lengths and different degradation amplitudes are plotted in Figs. 7 and 8. In plotting the graphs in Fig. 7 the parameters were chosen so that the response closely resembled the experimental response, and in each case the number of beat lengths chosen was the minimum necessary to yield a similar response to the graphs in Figs. 4.

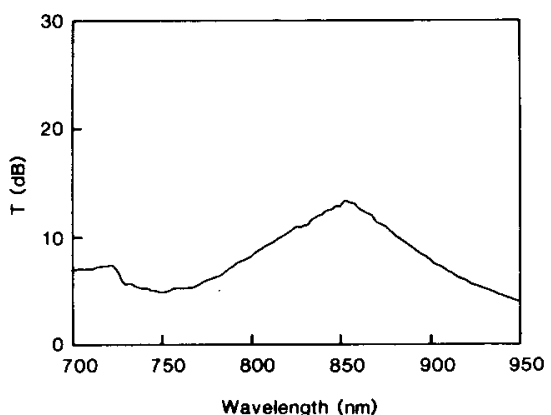

(a)

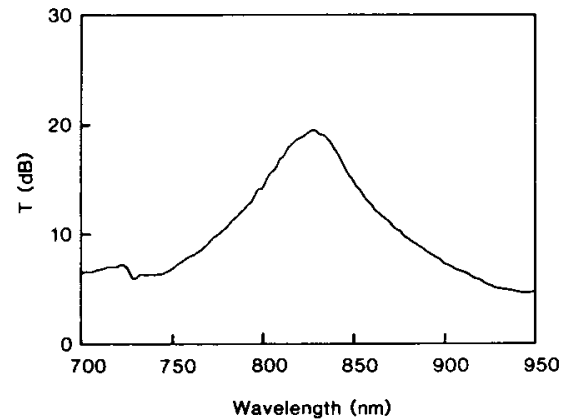

(b)



(c)

Fig. 4. Spectral transmission $T$ of the reflector for different levels of stress applied to the loop: (a) unrestrained loop lying freely on the bench; (b) loop compressed in the $G$-clamp; (c) loop compressed in the $G$-clamp under greater pressure than in (b). 


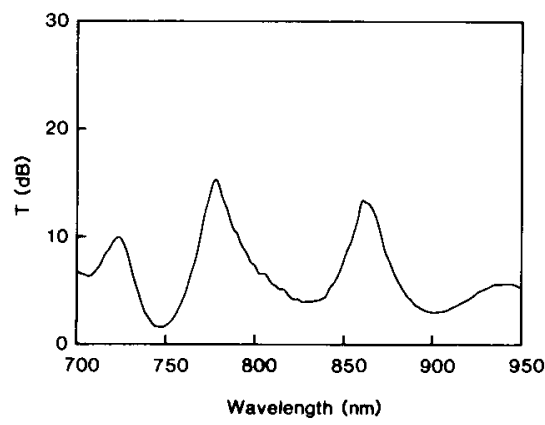

(a)

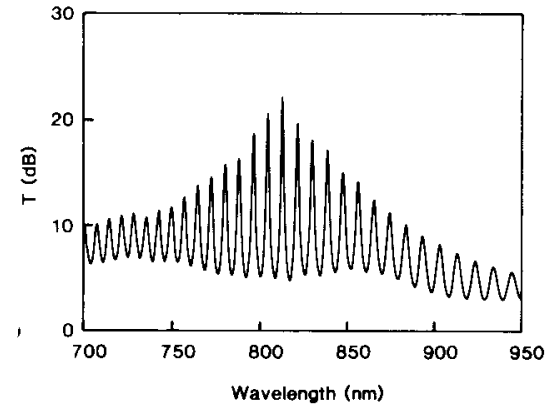

(c)



(b)

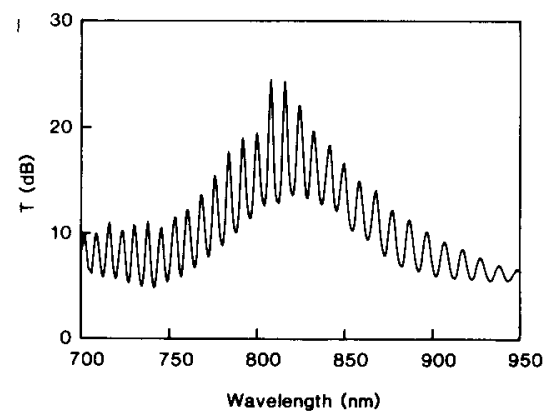

(d)

Fig. 5. Spectral transmission $T$ of the reflector with a length $L$ of HiBi fiber spliced into the loop: (a) $L=5 \mathrm{~cm}$; (b) $L=10.5 \mathrm{~cm}$; (c) $L=34 \mathrm{~cm}$; (d) same as (c) but with the loop lying in a different configuration on the bench.

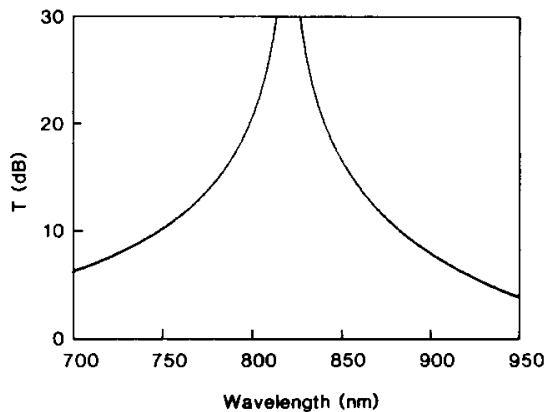

Fig. 6. Theoretical transmission $T$ of an ideal reflector using a coupler 3 -dB splitting point of $820 \mathrm{~nm}$ and a wavelength dependence of $\lambda^{5 / 2}$ in the coupling coefficient.
In plotting the graphs in Figs. 8 the number of beat lengths chosen corresponded directly to the number of beat lengths in the spliced-in HiBi fiber, while the degradation amplitude was chosen to give a similar overall response to Figs. 5.

\section{Discussion of Results}

In this work our postulate has been that the degradation in reflector performance is due to stray birefringence in the fiber loop. In general it is difficult to quantify the exact nature and extent of the birefringence present in any circuit. For example, if the loop is planar, a linear birefringence is introduced, the extent of which depends on the various curvatures present in the loop. ${ }^{7}$ If the loop is nonplanar, the birefringence becomes elliptical with perhaps some additional curvatures introduced.

Unknown levels of birefringence are also introduced through the packaging of the coupler and, in this case, through the protection required by the splice.

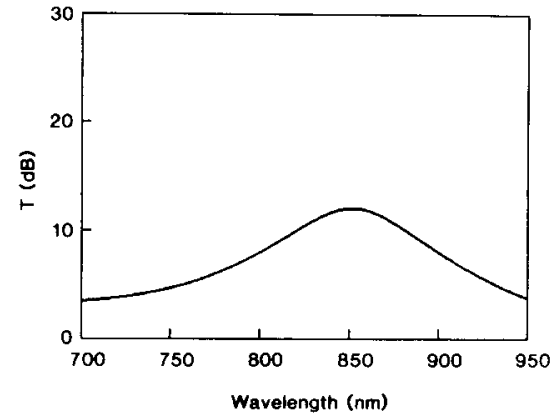

(a)

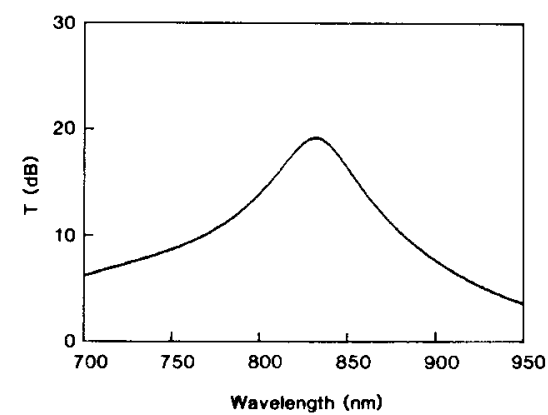

(b)

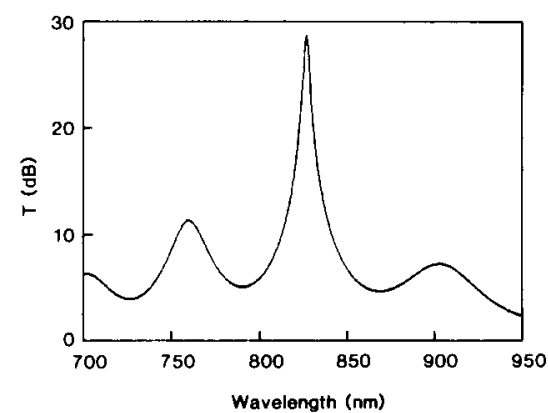

(c)

Fig. 7. Theoretical transmission $T$ of the reflector under the assumptions of Sec. IV with $N$ beat lengths in the loop at $820 \mathrm{~nm}$ and degradation amplitude: (a) $N=2.2$; D.A. $=0.3$; (b) $N=4.2$; D.A. $=0.05$; (c) $N=11.1$; D.A. $=0.3$. 


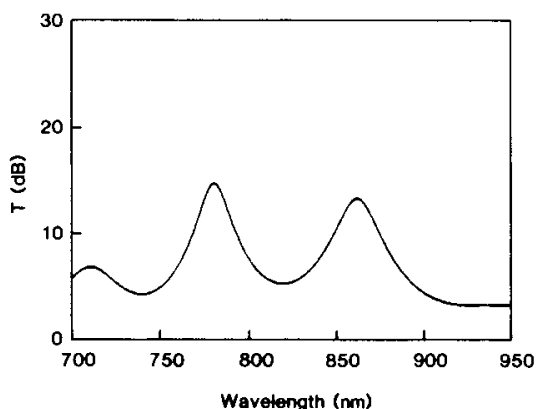

(a)



(c)

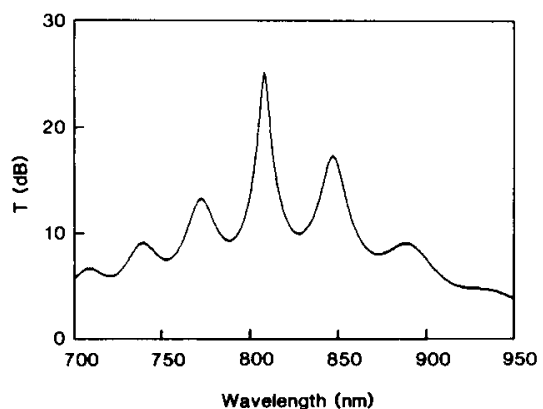

(b)

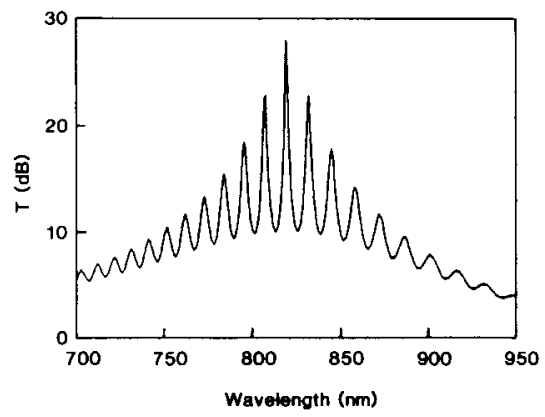

(d)

Fig. 8. Theoretical transmission $T$ of the reflector under the assumptions of Sec. IV with $N$ beat lengths in the loop at $820 \mathrm{~nm}$ and degradation amplitude: (a) $N=9.5$; D.A. $=0.3$; (b) $N=20.7$; D.A. $=0.1$; (c) $N=66.0$; D.A. $=0.4$; (d) $N=66.0$; D.A. $=0.1$.

To minimize the effects of such unknowns we introduced sections of known length of highly birefringent fiber with known birefringence into the loop. From the results obtained in these cases we confirm our postulate and can thereby explain the degradation of the normal reflector in terms of the birefringence of the loop.

From the above discussion it seems sensible to consider the figures in reverse order; i.e., loop with the $\mathrm{HiBi}$ fiber first. Figures $8(\mathrm{a})-(\mathrm{c})$ were plotted using the same number of fiber beat lengths as the experimental graphs of Figs. 5(a)-(c). Looking at these figures in detail, the responses look similar; from counting the number of oscillations ( $\alpha$ birefringence term $\left.\sin ^{2}[\pi N]\right)$ there is, however, more birefringence in the loop than can be accounted for by the fiber alone, and indeed in this set of experiments we have two protected splices to contend with rather than one.

In each of Figs. 8(a)-(c) the degradation amplitude factor (i.e., the factor $\sin ^{2}[2 R] \cos ^{2}[\gamma]$ ), which depends on the type of birefringence, was chosen arbitrarily to match the corresponding experimental responses in Figs. 5(a)-(c), since this factor is experimentally indeterminable. Figures 8(c) and (d) demonstrate the influence of this factor on the response curves for a fixed number of beat lengths, where in Fig. 8(c) the factor is 0.4 , and in Fig. 8(d) the factor is 0.1. Such a difference in the degradation factor can be due simply to the loop configuration on the bench as we found in Figs. 5(c) and (d).

Comparing Figs. 7 with Figs. 4, we clearly require birefringence in the loop to achieve a similar response. For the case with no extraneous strain on the loop Fig. $4(\mathrm{a})$, there is a birefringence equivalent to a minimum of 2.2 beat lengths in the loop at $820 \mathrm{~nm}$. Here the loop length was $\sim 50 \mathrm{~cm}$, and the birefringence due to a uniform curvature alone in a $125-\mu \mathrm{m}$ diam silica fiber should be of the order of 0.1 beat lengths. ${ }^{7}$ However, the stresses introduced by the splice and its protection should introduce a considerable birefringence in addition to that introduced by the coupler package. Intrinsic fiber birefringence can introduce as much as half of a beat length in this length of fiber, ${ }^{10}$ so that our estimate of 2.2 beat lengths in the loop is of the correct order.

\section{A. Effects of Losses}

In this paper we have consistently neglected the effects of fiber and coupler and splice losses, even though such losses can be considerable, especially when splicing a portion of unmatched $\mathrm{HiBi}$ fiber into the loop. Any losses in the reflector would reduce $\rho$ and $\tau$ by the same factor $F$, so that the real quantities $\rho^{\prime}$ and $\tau^{\prime}$ are related to the ideal (lossless) quantities $\rho$ and $\tau$ by

$$
\begin{aligned}
\rho^{\prime} & =F \rho, \\
\tau^{\prime} & =F \tau .
\end{aligned}
$$

Hence the logarithmic transmittivity plotted in the graphs is

$$
\begin{aligned}
T & =-10 \log _{10}\left(\tau^{\prime}\right) \\
& =-10 \log _{10}(\tau)-10 \log _{10}(F) .
\end{aligned}
$$

Since $F$ is expected to vary only slowly with wavelength, losses will not significantly distort the shape of the graphs other than by an approximately constant positive displacement. Hence the essential features of 
our graphs will be unaffected by such losses, and we are justified in neglecting them here.

\section{Conclusion}

In this paper we have considered the performance of one of the simplest fiber circuits, the Sagnac reflector. The response of this circuit is in practice degraded from the ideal, and this degradation is very dependent on any incidentally imposed external strains. Using the techniques of the Jones calculus we have derived an analytical expression for the response of the reflector [Eq. (13)] on the basis that there is birefringence present in the Sagnac loop. By measuring the spectral response of the reflector with various lengths of highly birefringent fiber spliced into the circuit we confirm the validity of the theory. We thereby confirm that the degradation in reflector performance is due to the presence of stress-induced birefringence.

Much of this birefringence is necessarily introduced in forming the loop. For example, the 0.1 birefringence beat lengths introduced into $50 \mathrm{~cm}$ of silica fiber by the action of uniformly bending it into a loop (Sec. V) will degrade the performance of the reflector by up to $10 \%$ (i.e., $90 \%$ reflectivity), even in the absence of the larger birefringences caused by coupler packaging, nonuniform loop curvature, the protection required by any splices present, and the intrinsic birefringence of the fiber. The analytical expressions derived should prove useful in the design of the Sagnac reflector, and the results demonstrate not only the importance of birefringence effects in determining the eventual performance of the reflector but also the applicability of the Jones calculus to the analysis of these effects within fiber circuits in general.

In this paper we have mainly considered the negative effects of birefringence in the Sagnac reflector. However, there may also be some positive aspects to such effects. For example, the wavelength response of the reflector with $\mathrm{HiBi}$ fiber spliced into the loop sug. gests the possibility of a new technique for fiber birefringence measurements which does not require the presence of polarizers, e.g., for the determination of fiber stress-optic coefficients. It also suggests the basis for the construction of a new type of stress-tunable single-mode fiber reflection filter, which could be used as a dichroic feedback element in fiber lasers.

The authors would like to thank C. D. Hussey for his help in writing this paper. A research studentship (TAB) was provided by the U.K. SERC.

\section{Reterences}

1. T. Bricheno and D. P. M. Chown "All-Fiber Signal Processing Components and Applications," in Technical Digest, Twelfth European Conference on Optical Communication, Barcelona (1986), pp. 421-424.

2. I. D. Miller, D. B. Mortimore, B. J. Ainslie, W. P. Urquhart, S. P. Craig, C. A. Millar, and D. B. Payne "New All-Fiber Laser," in Technical Digest, Optical Fiber Communication ConferenceSixth International Conference on Integrated Optics and Optical Fiber Communication (Optical Society of America, Washington, DC, 1987), paper WI3.

3. K. O. Hill, D. C. Johnson, F. Bilodeau, and S. Faucher "NarrowBandwidth Optical Waveguide Transmission Filters," Electron. Lett. 23, 465 (1987).

4. W. A. Shurcliff, Polarized Light-Production and Use (Oxford U.P., New York, 1962).

5. Ref. 4 , p. 28

6. Ref. 4 , p. 89.

7. J. N. Ross, "The Rotation of the Polarization in Low Birefringence Monomode Optical Fibers due to Geometric Effects," Opt. Quantum Electron. 16, 455 (1984).

8. F. P. Payne, C. D. Hussey, and M. S. Yataki, "Polarization Analysis of Strongly Fused and Weakly Fused Tapered Couplers," Electron. Lett. 21, 561 (1985).

9. M. P. Varnham, D. N. Payne, R. D. Birch, and E. J. Tarbox, "Single-Polarization Operation of Highly Birefringent Bow-tie Optical Fibers," Electron. Lett. 19, 246 (1983).

10. A. J. Barlow, D. N. Payne, M. R. Hadley, and R. J. Mansfield "Production of Single-Mode Fibers with Negligible Intrinsic Birefringence and Polarization Mode Dispersion," Electron. Lett. 17, 725 (1981). 